\title{
Temperature mapping, thermal diffusivity and subsoil heat flux at Kariavattom of Kerala
}

\author{
Tessy Chacko P and G Renuka \\ Department of Physics, University of Kerala, Kariavattom, Thiruvananthapuram, 695 581, India.
}

We have studied the soil and air temperature characteristics over a period of one year at Kariavattom of Kerala. Thermal diffusivity $\left(k_{\mathrm{s}}\right)$ of the soil has been calculated by range and lag methods and also from amplitudes and phase angles of first and second harmonics. The two methods lead to similar results. Diurnal soil heat flux and soil temperatures at different depths are modelled and found to be comparable with observations.

\section{Introduction}

Soil temperature is an important agrometeorological parameter. In general, the flow of heat through the soil is of considerable importance in plant growth. Extreme levels of temperature of soil as well as air affects plant life. Availability of water content causes variation in soil temperatures. The microclimate of soil is well understood through the study of heat flux.

The ground surface gets heated more during the day by intense solar radiation than the layers beneath, resulting in temperature gradient between the surface and subsoil on the one hand and surface and air layers near the ground on the other. Within the soil this causes heat flow downward as a thermal wave, the amplitude of which changes with depth. Estimation of heat flux from the soil temperature data can provide an understanding of the gain or loss of heat by the soil from the atmosphere. Many studies made earlier have been related to similar issues such as prediction of soil temperatures; heat storage variations; thermal diffusivity of the soil, etc. (Kelkar et al 1980; Chowdhury et al 1991; Lamba and Khambete 1991; Retnakumari et al 1996; Padmanabhamurty et al 1998 etc).

The objective of this study is to calculate the thermal diffusivity of the soil by different meth- ods. The soil heat flux and temperature of the soil at various depths are predicted. Section 2 gives the details of the data used. In section 3 the thermal diffusivity of the soil at different depths has been calculated by range and lag methods using the three-hourly measured soil temperatures of a wet (23rd August 2000) and dry (22nd January 2001) day. It is also estimated from the amplitudes and phase angles of first and second harmonics which are obtained by the harmonic analysis of monthly means of soil temperatures at 5, 10 and $20 \mathrm{~cm}$ depths. The soil and air temperature variations and soil heat flux at a depth of $2.5 \mathrm{~cm}$ are discussed. Sections 4 and 5 deal with the method of prediction of the soil temperature at different depths and its heat flux.

\section{Data}

The study utilises air temperatures at heights 50 , 122 , and $200 \mathrm{~cm}$ above the surface and soil temperature at depths $0,2.5,5,10,20$, and $50 \mathrm{~cm}$ for a period of one year from 1st April 2000 to 31st March 2001 recorded with soil thermometers installed at Kariavattom, Kerala. The experimental site is about $16 \mathrm{~m}$ above the mean sea level and belongs to soils of laterite landscape developed under tropical climate with alternate wet and dry

Keywords. Thermal wave; diffusivity; soil heat flux. 
seasons. The climate of Kerala is tropical, maritime and monsoonal in character.

Daily values of soil temperatures recorded at 0725 hours and 1425 hours IST have been averaged to obtain daily means. These means were then averaged to obtain normal soil temperature at different depths for the twelve standard months. The soil and air temperatures were also measured every three hours from 23rd to 25th August 2000 and 21st to 23rd January 2001. The above three days in January were dry and clear and those in August were rainy days. Some typical case studies based on these three-hourly temperature data are presented and discussed.

\section{Thermal diffusivity}

Thermal diffusivity $\left(k_{\mathrm{s}}\right)$ of the soil depends on soil moisture, soil porosity and conductivity of the soil particles. Of these, moisture content is the only short term variable for the given soil. Experimental determination of $k_{\mathrm{s}}$ of the soil is not easy in the field, hence it has been determined from the measured soil temperatures.

In the range method, the temperature range $R_{\mathrm{z}}$ at depth $z$ is given by (Chang 1968),

$$
R_{z}=R_{0} \exp \left\{-z\left(\frac{\pi}{k_{s} p}\right)^{1 / 2}\right\}
$$

where $R_{0}$ is the temperature range at the ground and $p$ is the oscillation period. In the lag method, the time lag for wave crest or trough to reach lower depths is given by (Oke 1978),

$$
t_{2}-t_{1}=\frac{z_{2}-z_{1}}{2}\left(\frac{p}{\pi k_{s}}\right)^{1 / 2}
$$

where $t_{1}$ and $t_{2}$ are the times at which the wave crest or trough reaches the depth $z_{1}$ and $z_{2}$.

Thermal diffusivity in different soil layers for the first two harmonics have been estimated from amplitude ratio and phase lag by assuming $k_{s}$ as constant. The monthly means of soil temperatures at 5, 10 and $20 \mathrm{~cm}$ depths of the year $(N=12)$ was subjected to Fourier analysis to obtain amplitudes and phase angles for all $\frac{N}{2}$ harmonics. The first two out of these harmonics have been considered (Retnakumari et al 1998). The amplitude of temperature fluctuation changes with depth $z$ and there is a shift of $z \sqrt{\frac{\pi}{p k_{s}}}$ in phase angle $\theta$ with depth, where $p$ is the period of fundamental cycle (Lamba and Sunita Bhandari 1998).

The average value of thermal diffusivity of the soil calculated by range method for a wet (23rd August 2000) and dry (22nd January 2001) days are $0.633 \times 10^{-6} \mathrm{~m}^{2} \mathrm{sec}^{-1}$ and $0.336 \times 10^{-6} \mathrm{~m}^{2} \mathrm{sec}^{-1}$ and by lag method are $0.668 \times 10^{-6} \mathrm{~m}^{2} \mathrm{sec}^{-1}$ and $0.307 \times 10^{-6} \mathrm{~m}^{2} \mathrm{sec}^{-1}$ respectively. The $k_{s}$ of soil is obviously affected by soil moisture. Oke (1978) indicated that $k_{s}$ of soil initially increases with the increase of moisture content and begins to decline when moisture content exceeds $20 \%$. The maximum of $k_{s}$ occurs at medium moisture values due to the added effect of vapour phase movement of moisture and heat transfer. The thermal diffusivity of the soil obtained by range and lag methods are in good agreement with each other and it changes with the degree of wetness of the soil.

The values of $k_{s}$ in different soil layers for first and second harmonics along with their consolidated values as computed from amplitudes and phase angles are presented in tables 1 and 2 . The combined effect of first and second harmonics give $k_{s}$ which show more consistency with that calculated by range and lag methods. Lamba and Sunita Bhandari (1998) indicated that bodily movement of moisture through soil can transfer large quantity of heat with it and the theory of heat conduction alone does not hold in the soil medium.

\section{Soil temperature}

In the case of diurnal forcing, the soil temperature $T_{s}(z, t)$ at depth $z$ has been modelled as (Padmanabhamurty et al 1998).

$$
T_{s}(z, t)=T_{a}+A_{0} \exp \left(\frac{-z}{D}\right) \sin \left(\Omega t-\frac{z}{D}\right)
$$

where $t$ is the time of observation reckoned from the zero reference value of temperature, $A_{0}$ is the half of the surface temperature amplitude, $T_{a}$ is the daily average surface temperature, $\Omega$ is the angular velocity of earth's rotation $\left(7.292 \times 10^{-5} \mathrm{rad} / \mathrm{sec}\right)$ and $D=\left(\frac{2 k_{s}}{\Omega}\right)^{1 / 2}$ is the damping depth for diurnal wave. The average value of $k_{s}$ obtained from range and lag methods is used.

Figures 1 and 2 show the diurnal variation of soil and air temperature for a dry (22nd January 2001) day. Large diurnal variations are significant up to $20 \mathrm{~cm}$ depth and up to screen level. The time of occurrence of lowest temperature at 
Table 1. Thermal diffusivity $\left(10^{-6} \mathrm{~m}^{2} \mathrm{sec}^{-1}\right)$ in different soil layers for first two harmonics as computed from amplitudes.

\begin{tabular}{rccc}
\hline $\begin{array}{c}\text { Soil depth } \\
\mathrm{cm}\end{array}$ & I Harmonic & II Harmonic & $\begin{array}{c}\text { Combined effect of } \\
\text { I and II harmonics }\end{array}$ \\
\hline $5-10$ & 0.030 & 0.059 & 0.089 \\
$10-20$ & 0.040 & 0.650 & 0.690 \\
$5-20$ & 0.034 & 0.187 & 0.221 \\
\hline
\end{tabular}

Table 2. Thermal diffusivity $\left(10^{-6} \mathrm{~m}^{2} \mathrm{sec}^{-1}\right)$ in different soil layers for first two harmonics as computed from phase angles.

\begin{tabular}{cccc}
\hline $\begin{array}{c}\text { Soil depth } \\
\mathrm{cm}\end{array}$ & I Harmonic & II Harmonic & $\begin{array}{c}\text { Combined effect of } \\
\text { I and II harmonics }\end{array}$ \\
\hline $5-10$ & 0.036 & 0.088 & 0.123 \\
$10-20$ & 0.058 & 0.245 & 0.303 \\
$5-20$ & 0.625 & 0.204 & 0.829 \\
\hline
\end{tabular}

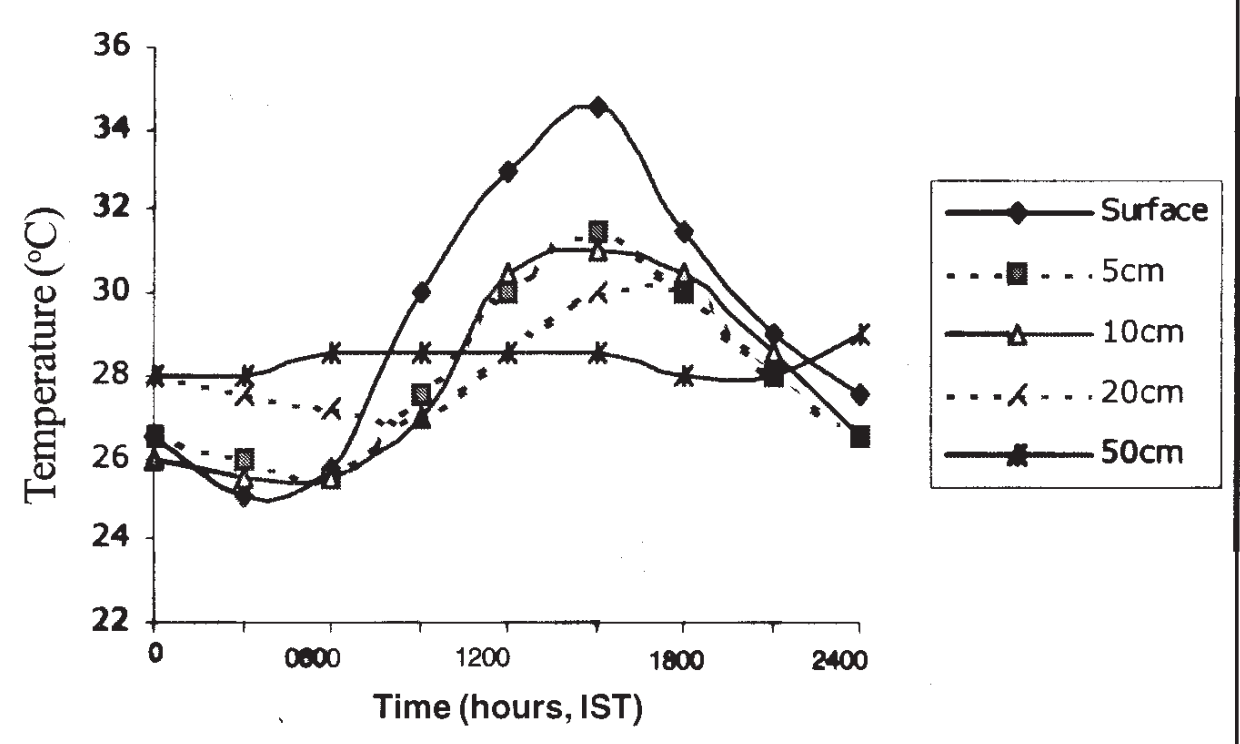

Figure 1. Diurnal variation of soil temperature on 22nd January, 2001.

heights of 200, 122 and $50 \mathrm{~cm}$ and within the soil up to $20 \mathrm{~cm}$ is generally near about sunrise. The range of soil temperature at surface is $9.5^{\circ} \mathrm{C}$. The diurnal soil temperature wave penetrates to $20 \mathrm{~cm}$ and $50 \mathrm{~cm}$ with ranges of $2.75^{\circ} \mathrm{C}$ and $0.5^{\circ} \mathrm{C}$. The temperature ranges at heights of 50, 122 and $200 \mathrm{~cm}$ are $9^{\circ} \mathrm{C}, 9^{\circ} \mathrm{C}$, and $8.5^{\circ} \mathrm{C}$ respectively. The diurnal wave penetrate to the atmosphere with only a slight lag and reduction in amplitude compared to soil medium. From the experi- mental data it is clear that during the day time, intense solar radiation is absorbed by the soil which warms the ground surface more than the layers beneath.

A comparison of the temperature on diurnal basis, between modelled and observed values has been presented in figure 3 . In the observed case, the temperature distribution at the surface, 5, 20, and $50 \mathrm{~cm}$ depths extend for a period of 36 hours starting from 0900 hours IST on 21st January 2001. The 


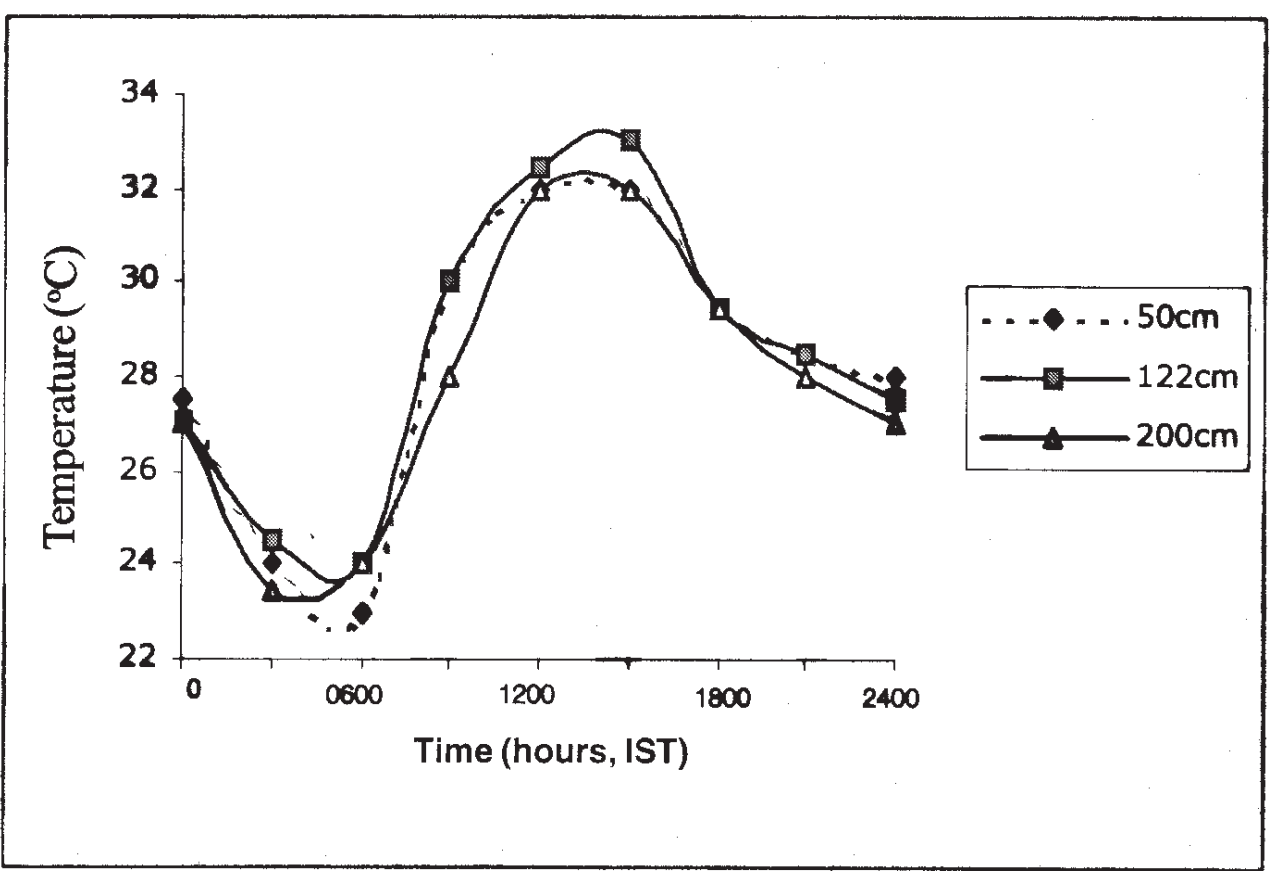

Figure 2. Diurnal variation of air temperature on 22nd January, 2001.
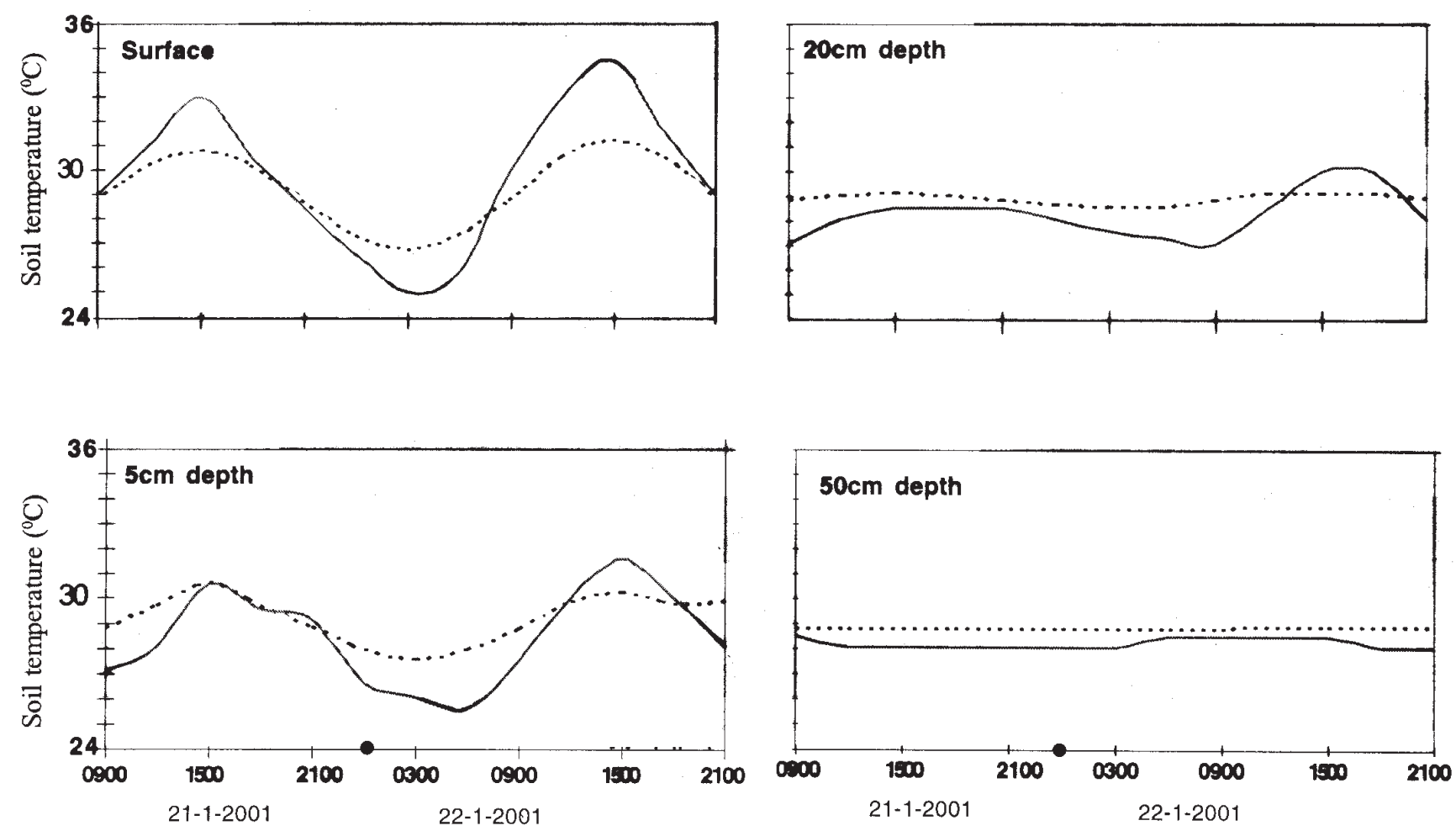

Time (hours, IST)

Time (hours, IST)

Figure 3. Variation of soil temperature from 0900 IST on 21st to 2100 IST on 22nd January 2001. 


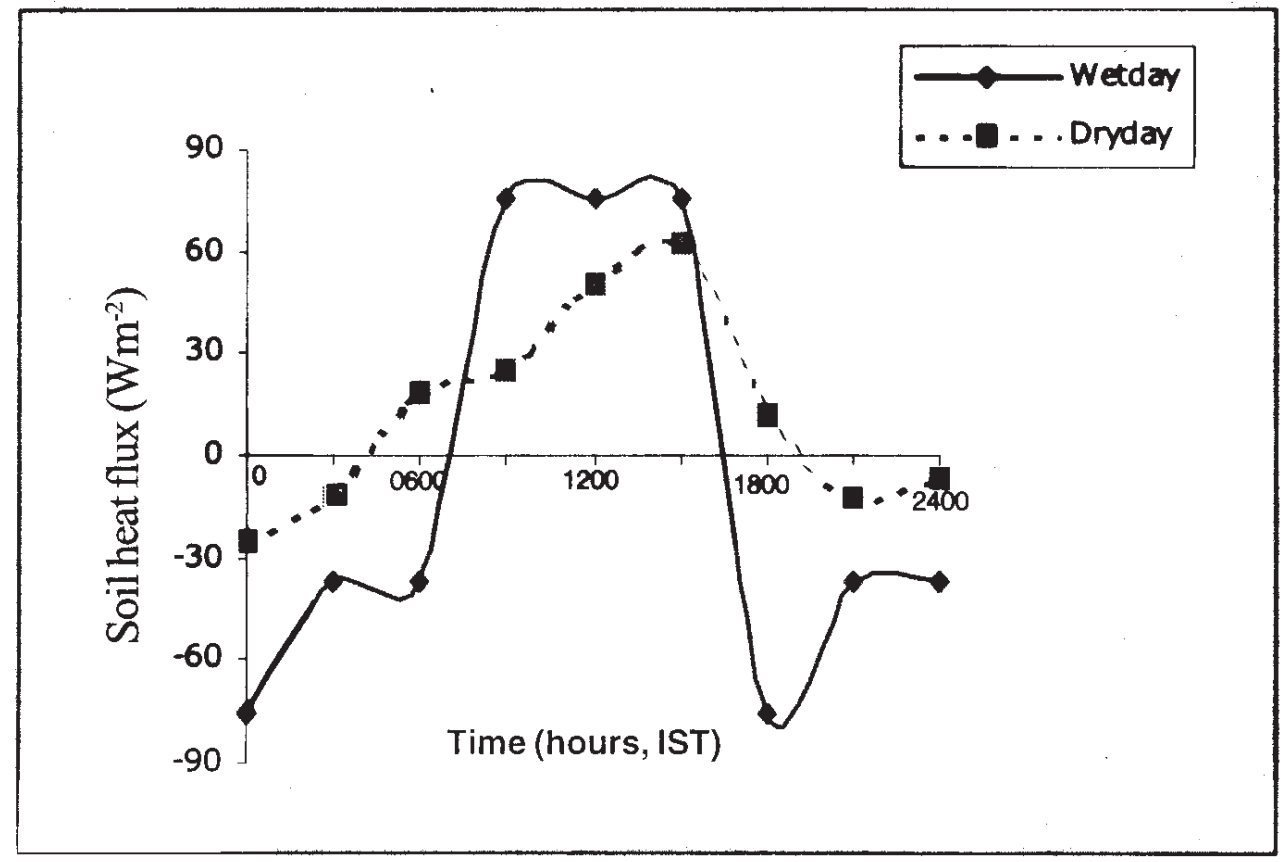

Figure 4. Diurnal variation of soil heat flux at $2.5 \mathrm{~cm}$ depth for a wet (23rd August, 2000) and dry (22nd January, 2001) day. (Negative and positive soil heat flux indicating a flux into and away from the surface).

wave nature of the temperature is deviated from the ideal sinusoidal behaviour as predicted by the model and reported by Garratt (1992). It can be seen that the model is slightly underpredicted in maximum and minimum surface temperatures. A deviation of $2^{\circ} \mathrm{C}$ is noticed in maximum and minimum surface temperature predictions when compared with actual measurements. The temperature of the ground surface dropped between 1500 and 1800 hours IST at a rate of $0.8^{\circ} \mathrm{C} / \mathrm{hr}$ and reaches maximum and minimum at rates of $1^{\circ} \mathrm{C} / \mathrm{hr}$ and $0.5^{\circ} \mathrm{C} / \mathrm{hr}$. The model could not accommodate such rapid changes in temperature resulting in a variation between observed and modelled temperatures. As a result, a difference occurred between observed and modelled temperature ranges. At $5 \mathrm{~cm}$ depth it is found that the model prediction of peak maximum temperature is in agreement with the observations. But the value of the minimum temperature is slightly underpredicted. The deviation between observed and modelled temperatures are found to be less at a depth of $20 \mathrm{~cm}$. The diurnal variation of temperature is very little at $50 \mathrm{~cm}$ depth. Overall, the model predictions are in agreement with the observations. It is noticed that the diurnal variation of surface temperature is decreasing as the depth of the soil increases.

\section{Soil heat flux}

The rate of heat flow in the soil depends on the strength of the mean temperature gradient. The soil heat flux $(G)$ is given by Oke (1978),

$$
G=K_{s} \frac{\partial T_{s}}{\partial z}
$$

where $\frac{\partial T_{s}}{\partial z}$ the vertical soil temperature and $K_{s}=C_{s} k_{s}$ the thermal conductivity. The volumetric heat capacity $C_{s}$ is computed as (Garratt 1992)

$$
C_{s}=\left(1-\eta_{s}\right) C_{s i}+\eta C_{w},
$$

where $\eta$ is the volumetric moisture content, $\eta_{s}$ is the saturation value, $C_{s i}$ is the volumetric heat capacity of the dry soil type $i$, and $C_{w}$ is the volumetric heat capacity of water. Soil moisture is determined by the gravimetric method and is converted into volumetric units. The values of $\eta_{s}, C_{s i}$ and $C_{w}$ are taken from Garratt (1992).

The soil heat flux at the depth of $2.5 \mathrm{~cm}$ with respect to the ground surface calculated using equation (4) has been modelled as (Padmanabhamurty et al 1998).

$$
G=C_{s}\left(k_{s} \Omega\right)^{1 / 2} A_{0} \sin \left(\Omega t+\frac{\pi}{4}\right)
$$

where $k_{s}$ and $A_{0}$ are determined from the observed soil temperature data.

Volumetric heat capacity of the soil on 23rd August $2000(\eta=0.32)$ and 22nd January 2001 $(\eta=0.13)$ computed using equation (5) are $2.89 \times$ 


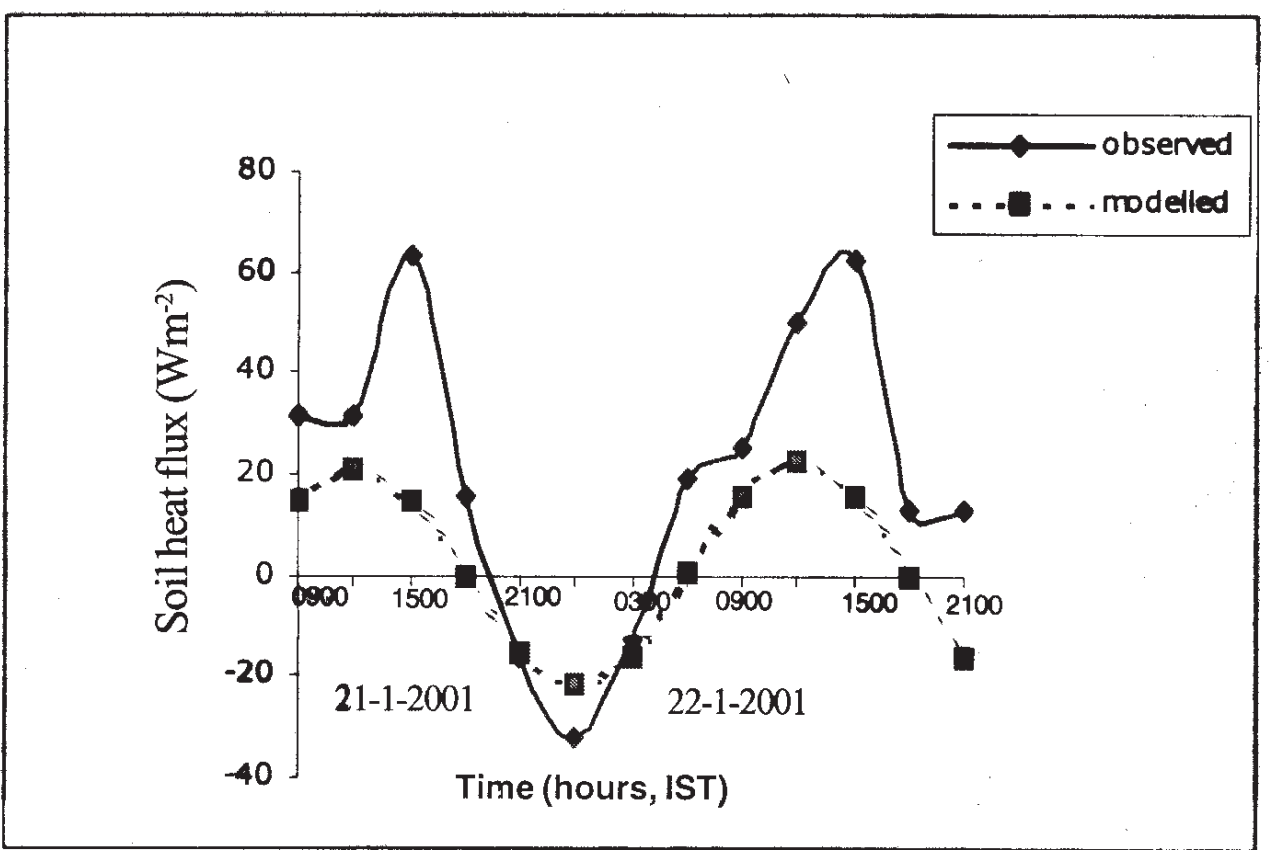

Figure 5. Variation of soil heat flux at $2.5 \mathrm{~cm}$ depth from 0900 IST on 21st to 2100 IST on 22nd January 2001.

$10^{6} \mathrm{Jm}^{-3} \mathrm{~K}^{-1}$ and $1.95 \times 10^{6} \mathrm{Jm}^{-3} \mathrm{~K}^{-1}$. Three hourly soil heat flux at $2.5 \mathrm{~cm}$ depth from 0000 to 2400 hours IST for the above mentioned wet $(\eta=0.32)$ and dry $(\eta=0.13)$ days are shown in figure 4 . In the wet day, the diurnal range of soil heat flux is low and 24-hour sum of the soil heat flux is negative which means the overall flow of heat from the soil to the atmosphere. The diurnal curve of soil heat flux in the dry day shows a well defined maximum around noon and 24-hour sum of the soil heat flux is positive. This is due to the rise of dryness of the soil and incoming solar radiation. Cloudiness changes the flow of heat into the soil. The thermal conductivity of the wet soil is high compared to dry soil. Diurnal curve on a wet day follows a more irregular pattern than on a dry day. This could be the result of variations in cloud cover and rainfall.

A comparison of soil heat flux on diurnal basis between modelled and estimated values has been presented in figure 5 . The wave nature of the heat flux is found to be deviated from the ideal sinusoidal behaviour as predicted by the model and reported by Garratt (1992). The modelled value of heat flux changes from positive to negative between 1500 and 1800 hours IST on 21st and from negative to positive between 0300 and 0600 hours IST on 22nd January 2001. Estimated heat flux shows these between 1800 and 2100 hours IST on 21st and between 0300 and 0600 hours IST on 22nd January 2001. The deviation found between modelled and estimated values could be less if the variable $C_{s}$ estimated directly at the experimental time.

\section{Conclusions}

- The thermal diffusivity of the soil obtained by range and lag methods are in good agreement with each other. The combined effect of the first two harmonics give more reliable values of thermal diffusivity (tables 1 and 2).

- The diurnal wave penetrates to atmosphere with only a slight lag and reduction in amplitude compared to soil medium (figure 2).

- The observed and modelled values of soil temperatures are in agreement with each other (figure 3$)$.

- The diurnal variation of heat flux is influenced by soil moisture and incoming solar radiation. In dry days the net flow of heat is directed into the soil and in rainy days the reverse takes place (figure 4). The estimated and modelled values of heat flux are comparable (figure 5).

\section{References}

Chang J 1968 Climate and agriculture (Chicago: Alderic Publishers)

Chowdhury A, Das H P and Pujari A D 1991 Subsoil temperature variation and estimation of soil heat flux at Pune; Mausam 42 357-360

Garratt J R 1992 The atmospheric boundary layer (Cambridge: Cambridge University Press)

Kelkar R R, Chivale V R and Dobey R C 1980 Observations of soil heat flux at Pune using a heat flux plate; Mausam 31 151-156

Lamba B S and Khambete N N 1991 Analysis of subsoil temperature at various depths by Fourier technique; Mausam 42 269-274 
Lamba B S and Sunita Bhandari 1998 Some aspects of thermal diffusivity for various soil layers in different harmonics; Mausam 49 255-258

Oke T R 1978 Boundary layer climate (New York: John Wiley and Sons)

Padmanabhamurty B, Amarlingeswara Rao and Rijula Mukherjee 1998 A preliminary analysis of soil temperature at five different sites; Indian J. Radio Space Phys. 27 199-206
Retnakumari K, Renuka G and Prasada Rao G S LHV 1998 Analysis of soil temperature in iso-hyperthermic temperature regime using Fourier technique; Indian J. Radio Space Phys. 27 207214

Retnakumari K, Renuka G and Thambi Raj S A 1996 Estimation of soil heat flux at Thiruvananthapuram during northeast monsoon and winter period; Mausam $\mathbf{4 7}$ 103

MS received 25 May 2001; revised 1 November 2001 